\title{
Ignorance is bliss: The role of observer expectation in dynamic spatial tuning of the attentional focus
}

\author{
LISA N. JEFFERIES AND SHAHAB GHORASHI \\ University of British Columbia, Vancouver, British Columbia, Canada \\ JUN-ICHIRO KAWAHARA \\ National Institute of Advanced Industrial Science and Technology, Tsukuba, Ibaraki, Japan \\ AND \\ VINCENT Di LOLLO \\ Simon Fraser University, Burnaby, British Columbia, Canada
}

\begin{abstract}
When two sequential targets (T1, T2) are inserted in an RSVP stream of distractors, perception of T2 is impaired at intertarget lags shorter than $700 \mathrm{msec}$. Paradoxically, this deficit disappears when T2 is presented directly after T1 (lag-1 sparing). Visser, Bischof, and Di Lollo (1999) found that lag-1 sparing occurs only when T1 and T2 are presented in the same stream. In contrast, Shih (2000) obtained lag-1 sparing with targets in separate streams. Four experiments addressed this inconsistency and revealed lag-1 sparing with targets in different streams, but only when observers had no foreknowledge of T1's location. We hypothesized that when T1 location is known, attention is focused narrowly on that stream; if T2 then appears in the other stream it is missed, and lag-1 sparing does not occur. When T1 location is not known, attention is focused broadly, encompassing both streams, and lag-1 sparing ensues.
\end{abstract}

Brief stimuli presented in rapid sequence may exceed the processing capability of the visual system. This gives rise to a deficit known as the attentional blink (AB), which is typically obtained when observers are required to identify two targets inserted in a stream of distractors presented in rapid serial visual presentation (RSVP). Identification accuracy for the second target is most impaired when the temporal lag between the two targets is short, and it improves as the intertarget lag is increased (Raymond, Shapiro, \& Arnell, 1992).

Theoretical accounts of the $\mathrm{AB}$ have relied on some form of limited attentional resource that is allocated to the leading target to the detriment of the trailing target. For example, in the interference model (Shapiro, Raymond, \& Arnell, 1994) resources are said to be allocated in large part to the first target, and in diminishing amounts to the ensuing items in the RSVP stream. Similar assumptions underlie the bottleneck models of Chun and Potter (1995) and Jolicour and Dell'Acqua (1998) in which the AB deficit is said to occur when the second target arrives while the resources at a high-level stage are preempted by the first target. On all these accounts, the $A B$ deficit is expected to be most pronounced when the second target is presented directly after the first, in the ordinal position known as lag 1.
Contrary to this expectation, a meta-analysis by Visser, Bischof, and Di Lollo (1999) revealed that in about half of the studies the $\mathrm{AB}$ deficit was much reduced or failed to occur when the second target was presented directly after the first. This yielded the characteristic U-shaped function of performance over lags often found in AB studies. Potter, Chun, Banks, and Muckenhoupt (1998) referred to this highly accurate performance at lag 1 as lag-1 sparing.

Lag-1 sparing has been ascribed to the sluggish closing of an attentional gate that serves the selective function of rejecting distractors and passing target items (Chun \& Potter, 1995; Shapiro \& Raymond, 1994). The gate is said to remain closed while the distractors prior to the first target are on display. Upon presentation of the first target, the gate is said to open rapidly, but to close sluggishly, thus allowing the next item in the stream (i.e., the lag-1 item) to gain access to processing resources along with the first target. If the trailing item happens to be the second target, both targets are processed together, and lag-1 sparing ensues. An important detail that emerged from Visser, Bischof, and Di Lollo's (1999) analysis was that lag-1 sparing occurs only when the two targets are presented at the same spatial location; it never occurs when they are presented at different locations. Such location specificity is exceptionally robust: Of the 41 relevant studies in which

L. N. Jefferies, ljefferies@psych.ubc.ca 


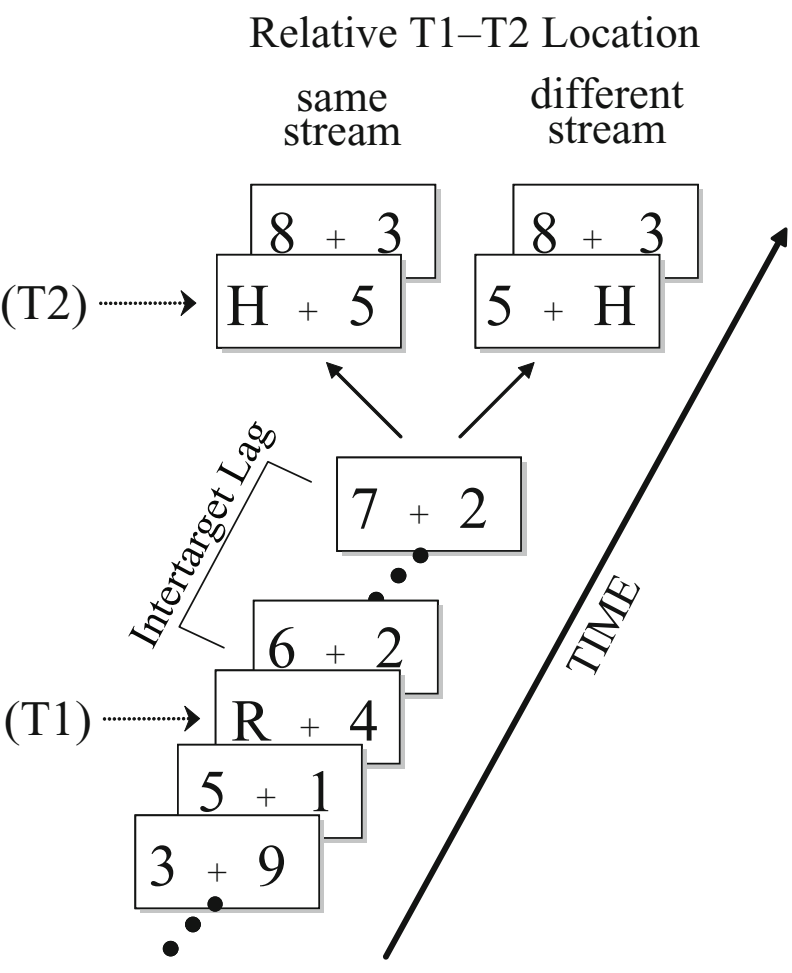

Figure 1. Schematic representation of the sequence of events within a trial in Experiment 1. Both the first and the second target (T1 and T2) could appear in either rapid serial visual presentation stream. At lag 1, the second target was presented directly after the first, without intervening distractors.

the two targets were presented at different locations, none revealed lag-1 sparing. This finding has been confirmed by Visser, Zuvic, Bischof, and Di Lollo (1999) in a study designed explicitly to examine the effect of the relative location of the two targets on lag-1 sparing.

An exception to this rule has been reported by Shih (2000), who found lag-1 sparing in an experiment in which the two targets were presented in different spatial locations. In Shih's experiments, observers viewed two RSVP streams, one on either side of fixation. The first target could appear in either the left- or the right-hand stream, and the second target could occur either in the same stream as the first target or in the opposite stream. Shih's results are clearly at odds with Visser, Bischof, and Di Lollo's (1999) finding that lag-1 sparing never occurs when the two targets are presented in different spatial locations. As discussed below, this discrepancy has significant implications for theories of the $\mathrm{AB}$. The present work was aimed at resolving the apparent inconsistency between Shih's results and the typical finding in the lag-1 sparing literature.

In our search for the critical factors underlying this discrepancy, we began by comparing Shih's (2000) study with similar studies in the $\mathrm{AB}$ literature. Two key factors emerged from this review: number of RSVP streams in the display and advance knowledge of the location of the first target. In the studies reviewed by Visser, Bischof, and Di Lollo (1999), the displays invariably contained only a single RSVP stream. In contrast, two concurrent streams were used in Shih's study. This gives rise to a confounding between number of streams and the observer's advance knowledge of the location of the first target. In the studies reviewed by Visser, Bischof, and Di Lollo (1999), the fact that the display contained only a single stream also meant that the observers always had advance knowledge of the location of the first target. In contrast, in Shih's study, observers never knew in which of the two streams the first target would appear. In the present study, we decoupled the roles of the observer's advance knowledge of the location of the first target and the presence or absence of a second RSVP stream as determinants of lag-1 sparing.

\section{EXPERIMENT 1}

Experiment 1 was designed to determine whether lag-1 sparing depends on the observer's advance knowledge of the first target's location, with the number of RSVP streams held constant. To this end, observers viewed two RSVP streams of digit distractors displayed one on each side of fixation. Two letter targets were inserted either in the same or in different streams. One group of observers did not know in advance in which stream the first target would appear. This group provides a direct replication of Shih's (2000) study. A second group knew in advance that the first target would always appear in a given stream.

\section{Method}

Participants. Thirty-four undergraduate students at the University of British Columbia participated for course credit. All reported normal or corrected-to-normal vision and were naive as to the purpose of the experiment. They were assigned randomly to one of two groups: the known group and the not-known group. One observer was removed from the not-known group for not following instructions.

Apparatus and Stimuli. A white fixation cross, $0.25^{\circ} \times 0.25^{\circ}$, was displayed in the center of the screen. The remaining stimuli consisted of white digits and letters, all of which subtended approximately $0.9^{\circ}$ vertically as viewed from a distance of approximately $57 \mathrm{~cm}$. The luminance of all stimuli was $90 \mathrm{~cd} / \mathrm{m}^{2}$, and the luminance of the black background was $2.3 \mathrm{~cd} / \mathrm{m}^{2}$.

Procedure. At the beginning of each trial, the fixation cross was presented in the center of the screen. The observers initiated each trial by pressing the space bar. The display sequence began with the presentation of two RSVP streams, one $1.75^{\circ}$ to the left of fixation, the other $1.75^{\circ}$ to the right. Each stream contained an equal number of digit distractors, and either 0,1 , or 2 letter targets, with the restriction that the two targets could not be the same letter. The number of leading distractors was the same in each stream on any given trial, but varied randomly between 8 and 14 across trials. The distractors were selected randomly from the digits $0-9$, with the restriction that the selected digit was not the same as either of the previous two digits. Two target letters, selected randomly from the English alphabet, with the exclusion of I, O, Q, and Z, were presented. The two targets could appear in either RSVP stream, depending on the condition. In the same condition, the two targets were presented in the same stream; in the different condition, they appeared in opposite streams. The first and the second target could appear in the left or in the right stream with equal probability. The display sequence on any given trial is illustrated schematically in Figure 1.

The observers were instructed to ignore the digit distractors and to identify both targets, in any order, by pressing the appropriate keys on the keyboard at the end of each trial. The second target was presented at one of six intertarget lags: lags 1, 3, 6, 9, 12, and 15, 
corresponding to stimulus onset asynchronies (SOAs) of 71, 214, $429,643,857$, and $1,071 \mathrm{msec}$. Intertarget lags occurred in random order and with equal frequency across trials. Distractors continued to be presented in the RSVP stream during the intertarget lag. The range of intertarget intervals used in the present experiment extends that used by Shih (2000). This was done because, in Shih's study, identification accuracy for the second target had not recovered completely, even at the longest intertarget lag of $571 \mathrm{msec}$.

The two RSVP streams were synchronized, with each item being displayed for approximately $14 \mathrm{msec}$, separated from the next item by approximately $57 \mathrm{msec}$. This resulted in a presentation rate of approximately 14 items/sec, which was the same as the rate in Shih's (2000) study. Each stream terminated with a single digit distractor, which acted as a mask for the second target.

The observers in one group, the not-known group, were instructed that on any given trial, the first target would appear unpredictably in either the left or the right RSVP stream. Half of the observers in the second group, the known group, were instructed that the first target would always be presented in the left RSVP stream. The remaining observers were instructed that the first target would always appear in the right RSVP stream.

In summary, three factors were manipulated in Experiment 1. The two within-subjects factors were intertarget lag $(1,3,6,9,12$, and 15 ) and second-target location (same or different stream, relative to the first target). The between-subjects factor was whether or not the observer knew in advance the location of the first target (known or not known).

\section{Results and Discussion}

In this and all subsequent experiments, only those trials in which the first target was identified correctly were included for analysis. This procedure is commonly adopted in $\mathrm{AB}$ experiments on the grounds that, on trials in which the first target is identified incorrectly, the source of the error is unknown, and thus its effect on second-target processing cannot be estimated. Correct identification of the first target averaged across lags and conditions was $76.5 \%$ for the known group and $67.2 \%$ for the not-known group. ${ }^{1}$

Figures 2A and 2B illustrate the percentage of correct second-target responses as a function of lag, relative second-target location, and observer's advance knowledge of the first target's location. The data were analyzed in a $2 \times 6 \times 2$ ANOVA comprising two within-subjects factors and one between-subjects factor. The within-subjects factors were relative second-target location (same or different stream relative to the first target) and lag $(1,3,6,9$, 12, and 15). The between-subjects factor was observer's advance knowledge of the first target's location (known or not known).

The analysis revealed a significant effect of lag $[F(5,155)=15.70, p<.001]$, and a significant interaction between lag and relative second-target location $[F(5,155)=11.69, p<.001]$. The three-way interaction among lag, relative second-target location, and observer's knowledge of the first target's location was also significant $[F(5,155)=4.30, p=.001]$. No other effects were significant.

In considering the outcome of the present work, it is well to be reminded that the issue of principal interest was Shih's (2000) finding that, contrary to the outcome of Visser, Bischof, and Di Lollo (1999), lag-1 sparing occurred

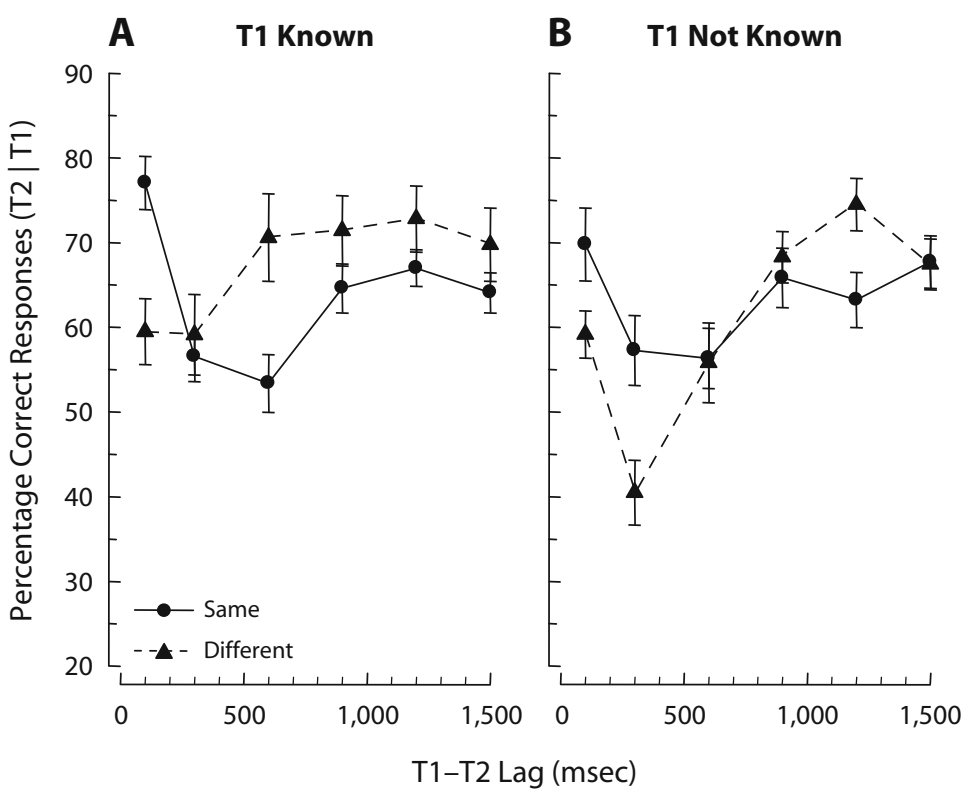

Figure 2. Mean percentages of correct identifications of the second target in Experiment 1 for those trials in which the first target was identified correctly (T2 T1). The displays consisted of two RSVP streams of digit distractors. In the same condition, the two letter targets were presented in the same RSVP stream; in the different condition, they were presented in opposite streams. Observers in the T1-known group (A) were given advance knowledge of the stream in which the first target would appear. Observers in the T1-not-known group (B) had no such knowledge. T1, first target; T2, second target; RSVP, rapid serial visual presentation. 
when the two targets were presented in different spatial locations. Therefore, while performance at the longer lags is not devoid of interest, the focus of the present work is on the incidence of lag-1 sparing, and therefore, on performance at the shorter lags. In this respect, it must be noted that interpretation of the three-way interaction revealed in the above analysis and illustrated in Figures 2A and 2B may involve effects at both the short and the long lags. Because the issue of principal interest in the present work was the incidence of lag-1 sparing, which is indexed by more accurate second-target identification at lag 1 than at lag 3, all further analyses were confined to lags 1 and 3 .

A follow-up ANOVA similar to the preceding analysis, except that it included only lags 1 and 3 , revealed a significant effect of relative second-target location $[F(1,31)=$ $15.35, p<.001]$ and lag $[F(1,31)=29.69, p<.001]$. As was the case in the overall analysis, the three-way interaction among lag, relative second-target location, and observer's advance knowledge of the first target's location was significant $[F(1,31)=12 \cdot 20, p=.001]$. No other effects were significant.

To single out the lag-1 sparing component in the threeway interaction effect revealed in the preceding ANOVA, we performed a simple simple main-effects analysis on the data for lags 1 and 3 in Figures 2A and 2B. The analysis showed that the effect of Lag was significant in both the same and different conditions in the not-known group $[F(1,60)=8.19, p=.006$, and $F(1,60)=18.22$, $p<.001$, respectively] and in the same condition of the known group $[F(1,60)=24.18, p<.001]$. Notably, the effect of lag was not significant in the different condition of the known group $[F(1,60)=0.02, p=.90]$. In light of this analysis, the three-way interaction can be interpreted as indicating that lag-1 sparing occurred in both the same and different conditions in the not-known group, but only in the same condition in the known group. This does not mean that effects at the longer lags did not contribute to the three-way interaction in the overall analysis; however, as noted above, the present work is concerned principally with lag-1 sparing and, therefore, with effects that occur at the shorter lags.

Before outlining a theoretical account of these results, two further issues need to be considered. The first relates to the possible effect of a systematic fixation bias, the second to the possible effects of practice. The possibility of fixation biases can be exemplified as follows. It is possible that when the location of the first target was known in advance, attention may have been deployed to that location not only covertly but also overtly by looking directly at that location, despite explicit instruction to maintain fixation at the central fixation cross. This raises the possibility that acuity differences across conditions might have played a role in performance above and beyond any covert allocation of an attention.

Had this been the case, however, the accuracy of second-target identification at lag 1 in the different condition (in which the targets were presented in opposite streams) should have been lower in the known condition (in which the observer was supposedly looking at the wrong stream) than in the not-known condition (in which the observer fixated either the center cross or one of the streams randomly).

This option can be checked by comparing performance at lag 1 in the different condition for the known and the not-known groups (Figure 2). Contrary to expectations based on fixation biases, the percentages of correct second-target responses in the two conditions were very similar (55.1\% for the known group and $59.1 \%$ for the not-known group) $[F(1,31)=0.005, p=.945]$. This strongly suggests that the observers maintained fixation in all conditions.

As for the possible effect of practice, it is conceivable that observers in the known group may have become more adept at attending to the designated stream (i.e., the stream that contained the first target) with extensive practice. In this case, the effect of knowledge of the first-target location would be confounded the effect of practice.

To check on this possibility, we compared mean firsttarget accuracy in the first $25 \%$ of the trials (when practice effects would be relatively small) with that in the last $25 \%$ of the trials (when practice effects would be relatively large), for the known and the not-known groups, collapsed over same and different conditions. The analysis revealed a slight (nonsignificant) improvement with practice overall $[F(1,40)=2.225, p=.145]$. But the improvement was not limited to the known group: The interaction effect between practice (i.e., first vs. last $25 \%$ of trials) and group was far from significant $[F(1,40)=12.48, p=.598]$. This strongly suggests that practice was not a major determinant of performance in the present work.

An attentional-gating account. An account of the present results as well as those of Shih (2000) and Visser, Bischof, and Di Lollo (1999) can be couched in terms of a modified version of the attentional gate described in the introduction. Such an attentional gate serves the selective function of rejecting distractors and allowing targets to gain access to a higher processing stage. To provide an adequate account, however, the description of the gate needs to be modified. Instead of a gate of fixed width, what is required is a gate whose spatial extent can be varied dynamically in order to optimize performance on the task at hand. Thus, depending on task demands, the gate may be set narrowly on a specific spatial location or broadly so as to encompass multiple locations.

The results for the four conditions illustrated in Figure 2 are explained by this model as follows. Given that the display contained two RSVP streams and that the observer knew in advance in which stream the first target would appear (Figure 2A), the gate was set to a relatively narrow opening, encompassing only the target's expected location. If the second target then appeared in the same location as the first, it fell within the ambit of the attentional gate, and lag-1 sparing ensued. If, however, the second target appeared in a different location, it fell outside the narrow confines of the gate, and lag-1 sparing did not occur. ${ }^{2}$

In contrast, if the observer had no advance knowledge of which stream contained the first target (Figure 2B), the setting of the attentional gate had to be widened so as to encompass both streams. Given this wider setting, the sec- 
ond target passed through the attentional gate regardless of whether or not it appeared in the same RSVP stream as the first target, and lag-1 sparing ensued.

This hypothesis is buttressed by the finding that accuracy for the first target was significantly greater when its location was known (76.5\%) than when it was not known $(67.2 \%)$. This is because when the location of the first target was known, the attentional gate was centered narrowly on that location. In contrast, when the location of the first target was not known in advance, the gate was set more widely in order to encompass both potential target locations and, therefore, attention was spread more diffusely.

One alternative attentional mechanism may be suggested in which the single attentional gate described above is replaced by two separate gates. In the known condition, there would be only a single gate, centered on the RSVP stream known to contain the first target; in the not-known condition there would be two gates, one centered on each RSVP stream. The two options (a dynamically expanding single gate or two separate gates) are functionally equivalent in that both can provide plausible accounts of the present results.

Performance at the shorter lags. Predictions from the attentional gating model can be assessed in greater detail by examining performance at each of the three shorter lags. Comparison of performance at lags 1, 3, and 6 in the different condition of the known and not-known groups (Figures 2A and 2B, segmented lines) shows that accuracy of second-target identification was approximately the same at lag 1 but substantially lower for the not-known group at lags 3 and 6 . The lower performance at lags 3 and 6 can be explained on two assumptions. The first assumption is based on the proposition that the $\mathrm{AB}$ deficit is caused by a disruption in second-target processing triggered by the distractors intervening between the first and second targets. This source of impairment was first proposed by Raymond et al. (1992), and was developed by Di Lollo, Kawahara, Ghorashi, and Enns (2005) as a comprehensive account of the $\mathrm{AB}$ deficit.

The second assumption is that visual processing is impaired during a spatial readjustment of the attentional focus. This assumption is based on the findings of Weichselgartner and Sperling (1987) that when the attentional focus is shifted from one RSVP stream to another, the items presented in the second stream within about $300 \mathrm{msec}$ of the attentional shift are often missed. According to the attentional-gating model, a spatial shift of attention was required in the different condition in the known but not in the not-known group. An account of how this may have led to the lower performance at lags 3 and 6 in the notknown group can be given as follows.

With reference to the findings of Weichselgartner and Sperling (1987), the attentional shift required in the known group caused the distractors directly preceding the second target to be missed. Not having been processed, those distractors could not trigger a disruption in secondtarget processing along the lines proposed by Raymond et al. (1992) and by Di Lollo et al. (2005). As a consequence, second-target performance was relatively less impaired. In contrast, no spatial shift of attention was re- quired in the not-known condition. This allowed the distractors preceding the second target to disrupt processing with consequently greater impairment in second-target identification.

We hasten to note that, while accounting for the lower performance at lags 3 and 6 , this reasoning cannot account for the finding that performance at lag 1 was about the same in the two groups. An indication as to why performance in the different-known and the different-notknown conditions differed at lag 3 but not at lag 1 may be gathered from Visser, Bischof, and Di Lollo's (1999) suggestion that lag-1 sparing and the $\mathrm{AB}$ are independent phenomena that arise at different stages of processing and have distinct neurophysiological correlates. More specifically, Visser, Bischof, and Di Lollo (1999) have suggested that lag-1 sparing may be based on the action of early filtering mechanisms whereas the $\mathrm{AB}$ may be mediated by domain-specific modules that become active later in the processing sequence. Applying Visser, Bischof, and Di Lollo's (1999) proposal to the present context would require a systematic investigation of the factors that influence performance at lags 1 and 3 in different ways. Although it is of potential interest, however, such an investigation is beyond the scope of the present work.

Relationship to earlier studies. From a general standpoint, the results of the present experiment replicate the conventional finding (e.g., Visser, Bischof, \& Di Lollo, 1999) that lag-1 sparing always occurs when the two targets are presented in the same location (Figures $2 \mathrm{~A}$ and $2 \mathrm{~B}$, same condition). The important new finding is that when the two targets are presented in different locations (Figures 2A and 2B, different condition), lag-1 sparing still occurs, but only when the observer does not have advance knowledge of the location of the first target.

The results for the not-known group, illustrated in Figure 2B, are virtually identical to those reported by Shih (2000, Figure 2A). The present study builds on Shih's findings by specifying the role of observer's knowledge of the first target's location, thereby permitting Shih's results and the results of Visser, Bischof, and Di Lollo's (1999) meta-analysis to be explained by a unitary set of principles. Furthermore, whereas in Shih's study accuracy for the second target never returned to lag-1 levels, the addition of longer lags in the present study allowed secondtarget accuracy to recover completely.

In contrast to the remarkable agreement between the present results and those of Shih (2000) and Visser, Bischof, and Di Lollo (1999), there are several studies that employed multiple RSVP streams and found no evidence of lag-1 sparing even though the observers had no advance knowledge of the first target's location (Dell'Acqua, Pascali, Jolicœur, \& Sessa, 2003; Holländer, Corballis, \& Hamm, 2005; Juola, Botella, \& Palacios, 2004; Kristjansson \& Nakayama, 2002; Peterson \& Juola, 2000). The lack of lag-1 sparing in these studies, however, is to be expected, based on differences in the SOA between successive items in the RSVP stream. Jefferies and Di Lollo (2006) have shown that whether lag-1 sparing occurs in multiple-stream studies depends critically on the duration of the SOA. Given that the two targets are presented 


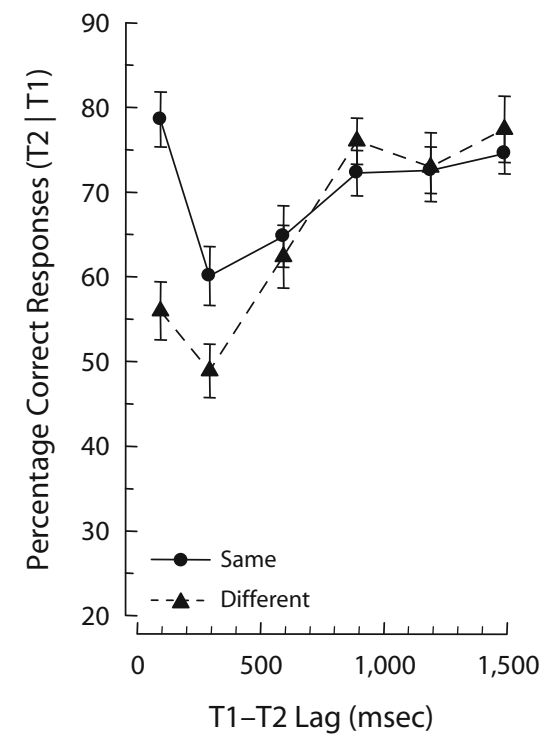

Figure 3. Mean percentages of correct identifications of the second target in Experiment 2 for those trials in which the first target was identified correctly (T2|T1). A single rapid serial visual presentation stream of digit distractors was presented randomly on either side of fixation with the location on the opposite side being blank. In the same condition, the two letter targets were presented in the same location; in the different condition, they were presented in opposite locations. T1, first target; T2, second target.

in different streams, lag-1 sparing occurs reliably when the SOA is shorter than approximately $100 \mathrm{msec}$, but not when it is $100 \mathrm{msec}$ or longer. Notably, in the above studies that failed to find lag-1 sparing, the SOAs ranged from 100 to $150 \mathrm{msec}$. In contrast, the SOA in the present study and in that of Shih, in which lag-1 sparing was obtained, was set at just over $70 \mathrm{msec}$.

In brief, all the available evidence from those studies in which lag-1 sparing was obtained (the present study, Shih's [2000] study, and Visser, Bischof, \& Di Lollo's [1999] meta-analysis) points to the same inference: Lag-1 sparing occurs reliably when the two targets are presented in the same location. When the two targets are presented in different locations, however, lag-1 sparing occurs only if the observers have no advance knowledge of the first target's location.

\section{EXPERIMENT 2}

Experiment 1 examined one of the two factors that emerged from our review of the literature, namely, the observer's advance knowledge of the first target's location. The outcome of Experiment 1 supplemented the findings of Visser, Bischof, and Di Lollo's (1999) meta-analysis by showing that lag-1 sparing occurs even when the two targets are presented in different locations, provided that the observer has no advance knowledge of the location of the first target. On the basis of this evidence, we concluded that the observer's advance knowledge is a critical determinant of lag-1 sparing. This conclusion cannot be regarded as definitive, however, because all the studies in which lag-1 sparing was not obtained when the two targets were presented at different locations contained only a single RSVP stream (Visser, Bischof, \& Di Lollo, 1999). This raises the possibility that the lag-1 sparing obtained in the different condition of the not-known group in Experiment 1 (Figure 2B, segmented line) may be contingent on the presence of two RSVP streams. Experiment 2 was designed to decouple the effects of advance knowledge of the first target's location and the number of RSVP streams.

In Experiment 2, only a single RSVP stream was presented, and the first and second targets, each followed by a mask, could appear unpredictably either within the stream or at a blank location on the opposite side of fixation. If the presence of two RSVP streams is essential to lag-1 sparing when the two targets are presented in different locations, then lag-1 sparing should not occur in the different condition of Experiment 2, even though the observer has no advance knowledge of the location of the first target. In a nutshell, the principal question in Experiment 2 was whether lag-1 sparing occurs under the following conditions: When the two targets are presented at different locations, the observer has no advance knowledge of the location of the first target, and there is no second RSVP stream.

\section{Method}

Participants. Eighteen undergraduate students at the University of British Columbia participated for course credit. All reported normal or corrected-to-normal vision and were naive as to the purpose of the experiment.

Procedure. The apparatus, stimuli, and procedure were the same as those in the not-known condition of Experiment 1, with the exception that instead of there being two RSVP streams, there was only a single stream that appeared with equal probability to the left or right of fixation. In addition, the first target could appear with equal probability either within the RSVP stream or at the blank location on the opposite side of fixation. The second target could then appear either in the same location as the first target or in the other location. When a target appeared in the blank location, it was always followed by a digit mask.

\section{Results and Discussion}

Correct identification of the first target averaged across lags and conditions was $70 \%$. Figure 3 illustrates the percentage of correct second-target responses as a function of lag and of relative second-target location. The data were analyzed in a $2 \times 6$ ANOVA comprised of two within-subjects factors: relative second-target location (same or different) and lag $(1,3,6,9,12$, and 15). The analysis revealed a significant effect of lag $[F(5,85)=$ $20.16, p<.001]$, a significant effect of relative secondtarget location $[F(5,85)=6.28, p=.023]$, and a significant interaction between lag and relative second-target location $[F(5,85)=9.49, p<.001]$. The significant interaction effect, coupled with the graphical evidence in Figure 3 indicates that performance in the same and different conditions differed at lags 1 and 3 , but not at the longer lags. To check on the incidence of lag-1 sparing, separate $t$ tests were performed in which the mean second-target 
accuracy at lags 1 and 3 were compared in the same and the different conditions separately. The analyses revealed that performance at lag 1 was significantly higher than at lag 3 in both the same condition $[F(1,17)=17.98, p=$ $.001]$ and in the different condition $[F(1,17)=4.80, p=$ $.043]$, confirming that lag-1 sparing occurred whether the two targets were presented in the same location or in different locations.

The results of Experiment 2 provide an unambiguous answer to the initial question: Does lag-1 sparing occur when the two targets are presented at different locations, the observer has no advance knowledge of the location of the first target, and there is no second RSVP stream. From Figure 3 and from the statistical analyses, it is clear that lag-1 sparing occurred when the two targets were presented at different locations, even though the display contained only a single RSVP stream. It follows that the presence of a second RSVP stream is not necessary for lag-1 sparing; what matters is that the observers have no advance knowledge of the location of the first target. This finding is entirely consistent with the attentional gating hypothesis outlined above. When the location of the first target is not known in advance, the gate is set to encompass both potential target locations. This allows the second target to pass through the gate regardless of whether it appears at the same location as the first target or at a different location.

A comparison of Experiment 2 with the not-known condition of Experiment 1 (Figures 3 and 2B) permits an assessment of the relative prominence of endogenous and exogenous factors in setting the width of the attentional gate. It is well to be reminded that, given that the location of the first target is not known, the observer's ideal strategy would be to optimize the width of the attentional gate so as to encompass both potential locations. If exogenous factors play a role, the presence of a second RSVP stream would provide an additional anchor point to mediate accurate setting of the gate's width. In this case, the magnitude of lag-1 sparing should be greater in the not-known condition of Experiment 1, where the display contained two RSVP streams, than in Experiment 2, where the display contained only a single stream. If, on the other hand, the width of the gate can be set entirely endogenously, then the presence or absence of a second RSVP stream should have no effect on the setting of the gate and hence on the magnitude of lag-1 sparing. It goes without saying that these considerations apply only to the different condition.

We examined this issue by comparing the magnitude of lag-1 sparing (i.e., performance differences between lags 1 and 3 ) in the different condition of Experiment 2 and the different condition of the not-known group in Experiment 1. The $2 \times 2$ ANOVA consisted of one between-subjects factor (experiment: the different conditions in Experiment 2 and the not-known condition of Experiment 1) and one within-subjects factor (lag: 1 and 3). The analysis revealed a significant effect of lag $[F(1,32)=34.05, p<.001]$ and a significant interaction between experiment and lag $[F(1,32)=6.87, p=.013]$. The effect of experiment was not significant $[F(1,32)=0.35, p=.559]$.
The outcome of this analysis indicates that the magnitude of lag-1 sparing was significantly greater when the display contained two RSVP streams than when it contained only one. This strongly suggests that exogenous factors, while perhaps not as prominent as endogenous factors, do play a role in setting the width of the attentional gate. It must be emphasized, however, that exogenous factors, while important, are not necessary for lag-1 sparing. This is revealed by the finding of a significant amount of lag-1 sparing in the different condition of Experiment 2, in which a wider setting of the attentional gate could be mediated only by endogenous factors.

\section{EXPERIMENT 3}

Experiment 3 was designed to pursue the finding that the magnitude of lag-1 sparing in the different condition in Experiment 2 was significantly smaller than in the corresponding condition in Experiment 1 (segmented line in Figure 3 vs. segmented line in Figure 2B). According to the attentional gating hypothesis, this difference can be explained in terms of the factors that govern the width of the gate. Because in neither case did observers have advance knowledge of the location of the first target, the optimal strategy was to maintain a gate setting sufficiently wide to encompass both potential target locations. This strategy, however, might have been more easily implemented in Experiment 1 than in Experiment 2. This is because, in Experiment 1, the display contained two RSVP streams, which provided anchoring points that enabled the observers to set and maintain a sufficiently wide attentional gate. In this case, endogenous and exogenous factors worked in conjunction with one another in setting the spatial extent of the gate. In contrast, in Experiment 2, the display contained only a single stream, and it is known that under these display conditions, attention is drawn in an obligatory fashion to the location of the RSVP stream (Kawahara, 2003; Visser, Bischof, \& Di Lollo, 2004). In this case, endogenous and exogenous factors worked in opposition to one another: The former would make for a gate of an appropriate width whereas the latter would make for a gate more narrowly confined to the RSVP stream. In Experiment 2 this would have resulted in a somewhat narrower gate that failed to encompass the blank location, at least on some trials. If it is indeed the case that lag-1 sparing occurs only when the attentional gate encompasses the location of the second target, it follows that the magnitude of lag-1 sparing would be greater in Experiment 1 than in Experiment 2.

We tested this hypothesis in Experiment 3 by introducing a second RSVP stream consisting of a series of frames of random dots. Our intent was to establish a second anchor point to provide exogenous inputs that could be used to expand the width of the attentional gate. We reasoned that each frame in the random-dot stream would trigger an onset transient, thereby providing exogenous stimulation. Being meaningless, however, this random-dot stream would avoid any possible interference by endogenous factors that might accompany a stream of meaningful items, as might have been the case in Experiment 1. It 


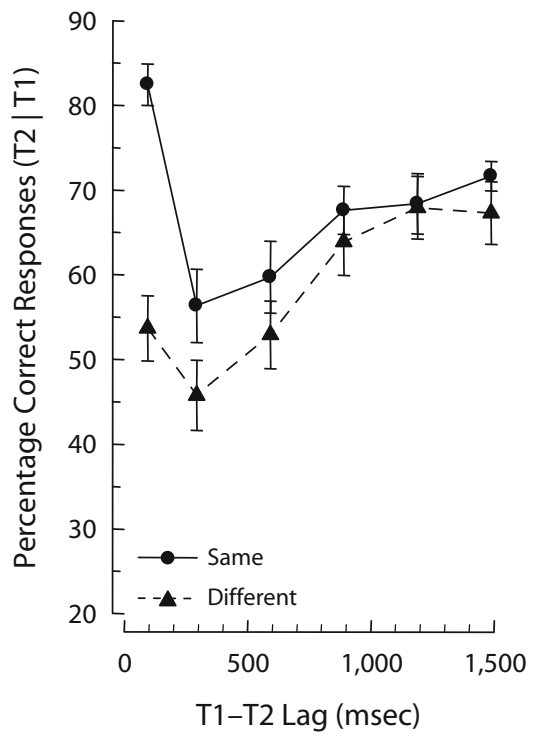

Figure 4. Mean percentages of correct identifications of the second target in Experiment 3 for those trials in which the first target was identified correctly (T2 $\mid \mathrm{T} 1)$. The displays consisted of two rapid serial visual presentation streams, one of digit distractors, the other of random-dot patterns. In the same condition, the two letter targets were presented in the same stream; in the different condition, they were presented in opposite streams. T1, first target; T2, second target.

goes without saying that the random-dot stream differed from the digit stream in aspects beyond meaningfulness. For example, the two streams differed in target-distractor similarity, structural composition, and number of parts in each item, to name but a few potential differences. In what follows, we use "meaningfulness" as a shorthand term to encompass all these actual and potential differences between the two distractor streams.

\section{Method}

Participants. Sixteen undergraduate students from the University of British Columbia participated for course credit. All reported normal or corrected-to-normal vision and were naive as to the purpose of the experiment.

Procedure. The apparatus, stimuli, and procedure were identical to those of the not-known condition in Experiment 1 with the exception that one of the two RSVP streams of digits was replaced with a stream of random-dot patterns that differed randomly on each frame. Each pattern contained 10 dots distributed randomly over a square area of $0.9^{\circ}$ centered $1.75^{\circ}$ to the left or right of fixation. The two targets could appear in either RSVP stream and were always followed by a digit mask.

\section{Results and Discussion}

Correct identification of the first target averaged across lags and conditions was $81 \%$. Figure 4 illustrates the proportion of correct second-target responses as a function of lag and of intertarget spatial relationship. The data were analyzed in a $2 \times 6$ ANOVA comprised of two withinsubjects factors: intertarget spatial relationship (same or different) and lag (1, 3, 6, 9, 12, and 15). The analysis revealed significant effects of intertarget spatial relationship $[F(1,15)=15.41, p=.001]$ and $\operatorname{lag}[F(5,75)=17.57$, $p<.001]$. The interaction between lag and intertarget spatial relationship was also significant $[F(5,75)=12.91$, $p<.001]$. The significant interaction effect, coupled with the graphical evidence in Figure 4 indicates that performance in the same and different conditions differed at lags 1 and 3 , but not at the longer lags.

To check on the incidence of lag-1 sparing, separate $t$ tests were performed between lags 1 and 3 in the same and the different conditions separately. The analyses revealed that performance at lag 1 was significantly higher than at lag 3 in both the same condition $[F(1,15)=44.09$, $p<.001]$ and the different condition $[F(1,15)=6.35$, $p=.024]$, confirming that lag-1 sparing occurred not only when the two targets were presented in the same location, as is commonly found in $\mathrm{AB}$ experiments, but also when they were presented in different locations.

The main objective of Experiment 3 was to determine whether providing a second anchor point in the form of a meaningless RSVP stream facilitates the expansion of the attentional gate beyond the width established in Experiment 2. A wider setting of the gate should cause the magnitude of lag-1 sparing in the different condition of Experiment 3 to be greater than that in Experiment 2. To check on this option, the results for lags 1 and 3 of the different condition of Experiments 2 and 3 were analyzed in a $2 \times 2$ ANOVA comprising one between-subjects factor (experiment: 2 and 3 ) and one within-subjects factor (lag: 1 and 3). The analysis revealed a significant effect of lag $[F(1,30)=9.20, p=.005]$, but no significant interaction between lag and experiment $[F(1,30)=.322, p=.575]$. On the basis of this analysis it can be concluded that the magnitude of lag-1 sparing was no greater in Experiment 3 than in Experiment 2. In terms of the gating hypothesis, this means that the presence of a second, meaningless, RSVP stream does not provide a useful anchor point for setting the width of the attentional gate and, therefore, that exogenous factors play only a minimal role.

In contrast, an equivalent comparison between Experiment 3 and the not-known group in Experiment 1 revealed that the magnitude of lag-1 sparing in the different condition was significantly smaller in Experiment 3 . This was confirmed in a subsidiary 2 (lag: 1 and 3 ) $\times 2$ (experiment: 3 and 1) ANOVA, which revealed a significant effect of $\operatorname{lag}[F(1,30)=38.09, p<.001]$ and, critically, a significant interaction between lag and experiment $[F(1,30)=$ $6.17, p=.019]$. This provides further confirmation that a stream of random-dot patterns does not provide a suitable anchor point for setting the width of the attentional gate.

The finding of primary interest that emerges from these analyses is that the magnitude of lag-1 sparing in the different condition was the same in Experiments 2 and 3, but was greater in the not-known group in Experiment 1 than in either Experiment 2 or 3 . This strongly suggests that with respect to providing an anchor point for setting the width of the attentional gate, a stream of random dots is no better than no stream at all. It may be suggested that the random-dot stream in Experiment 3 may not have been functionally different from the blank stream in Experiment 2. Specifically, the onset of a random-dot frame may not have been more effective in attracting attention than 
the onset of a blank frame. This hypothesis, however, is not supported by the evidence in the literature on attentional capture, notably the finding that, unlike the onset of a blank frame, the sudden onset of a pattern - whether random or not-attracts attention automatically (Theeuwes \& Burger, 1998; Yantis \& Jonides, 1984, 1996). This argues in favor of the hypothesis that the random-dot RSVP stream in Experiment 3 was an effective exogenous cue.

Why was the magnitude of lag- 1 sparing less in the different condition of Experiments 2 and 3 than in the corresponding condition of the not-known group in Experiment 1 ? Or, in terms of the gating hypothesis, why could the gate be set to a wider opening in Experiment 1 than in either Experiment 2 or 3 ? An answer to this question may be found in the balance of meaningfulness between the streams in the two potential target locations. In Experiment 1, both streams contained meaningful items and, therefore, meaningfulness was balanced across the two locations. In Experiments 2 and 3, on the other hand, only one of the streams contained meaningful items, resulting in an imbalance across the two locations. It is conceivable that, because of this imbalance, attention was focused more narrowly on the meaningful stream with a corresponding reduction in the probability that the attentional gate encompassed both potential target locations on any given trial.

\section{EXPERIMENT 4}

This possibility was examined in Experiment 4 by presenting two RSVP streams of random-dot patterns. If balance in the meaningfulness of the two streams is the critical factor underlying the greater magnitude of lag-1 sparing in Experiment 1 than in Experiments 2 and 3, then the results of Experiment 4, in which the two streams are balanced, should be comparable to those of Experiment 1. In order to provide a complete replication of Experiment 1, Experiment 4 included a known group as well as a not-known group. According to the attentional gating hypothesis, no lag-1 sparing should be in evidence in the different condition of the known group in Experiment 4, paralleling the results in the corresponding condition of Experiment 1.

\section{Method}

Participants. Thirty-four undergraduate students from the University of British Columbia participated for course credit and were randomly assigned to either the known or the not-known group. All reported normal or corrected-to-normal vision and were naive as to the purpose of the experiment.

Procedure. The apparatus, stimuli, and procedure were identical to those of Experiment 1 with the exception that both the RSVP streams of digits were replaced with streams of random-dot patterns that differed randomly on each frame. Each pattern contained 10 dots distributed randomly over a square area of $0.9^{\circ}$ centered $1.75^{\circ}$ to the left and right of fixation. On any given frame, the two randomdot patterns were different from one another. The two targets were always followed by a digit mask.

The observers in the not-known group were instructed that on any given trial, the first target would appear unpredictably in either the left or the right RSVP stream. Half of the observers in the second group, the known group, were instructed that the first target would always be presented in the left RSVP stream. The remaining observ- ers were instructed that the first target would always appear in the right RSVP stream.

\section{Results and Discussion}

Correct identification of the first target averaged across lags and conditions was $86 \%$ for the known group and $83 \%$ for the not-known group. Figures 5A and 5B illustrate the percentage of correct second-target responses as a function of lag, relative second-target location, and observer's advance knowledge of first target's location. The present experiment was designed as a replication of Experiment 1 with the exception that the items in the RSVP stream were meaningful in Experiment 1, but meaningless in the present experiment. Comparison of Figures 5A and $2 \mathrm{~A}$ reveals somewhat different patterns of results for the known group of Experiments 1 and 4. These differences are in themselves intriguing and worthy of further investigation. For the present purposes, however, the important consideration is that lag-1 sparing did not occur in the different condition of the known group in either Experiment 1 or 4 . In contrast, comparison of Figures 5B and 2B shows that the results of Experiment 4 replicated the two critical findings of Experiment 1. First, lag-1 sparing invariably occurs when the two targets are presented in the same location. Second, and more important, lag-1 sparing also occurs when the two targets are presented in different locations, but only when the observer does not have advance knowledge of the location of the first target.

The results of Experiment 4 were analyzed in an overall $2 \times 6 \times 2$ ANOVA comprising two within-subjects factors and one between-subjects factor. The within-subjects factors were relative second-target location (same or different stream relative to the first target) and lag $(1,3,6,9$, 12 , and 15). The between-subjects factor was observer's advance knowledge of the first target's location (known or not known). The analysis revealed significant effects of lag $[F(5,160)=40.95, p<.001]$, relative second-target location $[F(1,32)=9.17, p=.005]$, and observer's advance knowledge of the first target's location $[F(1,32)=$ $5.14, p=.03]$. There were also significant interaction effects between relative second-target location and lag $[F(5,160)=36.40, p<.001]$ and among lag, relative second-target location, and observer's advance knowledge of the first target's location $[F(5,160)=5.89, p=.001]$. No other effects were significant.

The graphical evidence in Figures 5A and 5B strongly suggest that the three-way interaction arises from differences in performance between the known and not-known groups at the shorter lags because performance at the longer lags was comparable in the two groups. Notably, lag-1 sparing was in evidence in the results of the different condition of the not-known group (Figure 5B) but not in the corresponding condition of the known group (Figure 5A), paralleling the findings of Experiment 1 . The presence of lag-1 sparing in the different condition of the not-known group in Experiment 4 was confirmed by a $t$ test showing that accuracy of second-target identification was higher at lag 1 than at lag $3[F(1,16)=49.70, p<.001]$. Similarly, the absence of lag-1 sparing in the different condition of the known group was confirmed by a $t$ test showing that 
second-target identification was, if anything, lower at lag 1 than at lag $3[F(1,16)=1.21, p=.286]$. The outcome of these $t$ tests, coupled with the graphical evidence in Figures $5 \mathrm{~A}$ and $5 \mathrm{~B}$, justifies the inference that lag-1 sparing does occur in the different condition, but only when the observer has no advance knowledge of the location of the first target.

This pattern of results is entirely consistent with the hypothesis that balance in the meaningfulness of the two streams is the critical factor underlying the greater magnitude of lag-1 sparing in the different condition of Experiments 1 and 4 (Figures 2B and 5B, not-known groups) than in the different condition of Experiments 2 and 3 (Figures 3 and 4). To wit, in Experiments 1 and 4 the two RSVP streams were balanced with respect to meaningfulness: Both streams were meaningful in Experiment 1 and meaningless in Experiment 4. In contrast, in Experiments 2 and 3, the displays were unbalanced because only one potential target location contained a meaningful RSVP stream; the other location was either empty (Experiment 2) or contained a stream of meaningless items (Experiment 3).

A subsidiary analysis confirmed that the magnitude of lag-1 sparing in the different condition of the not-known group of Experiment 4 did not differ significantly from that in the corresponding condition of Experiment 1. The analysis involved a comparison between lags 1 and 3 for the different condition of the not-known groups in Experiments 1 and 4. The ANOVA comprised one betweensubjects factor (experiment: 1 or 4) and one within-subjects factor (lag: 1 or 3 ). The outcome revealed a significant effect of lag $[F(1,31)=88.86, p<.001]$ but no other significant effects. Notably, the interaction between experiment and lag was not significant $[F(1,31)=0.179, p=$ .675], attesting to the equivalent magnitude of lag-1 sparing in the two experiments. In contrast, the magnitude of lag-1 sparing was greater in the different condition of the not-known group in Experiment 4 than in the corresponding condition of either Experiment 2 or Experiment 3. This was confirmed by two additional analyses. The first involved a comparison between lags 1 and 3 for the different condition of the not-known group in Experiment 4 with the different condition in Experiment 2. The analysis revealed a significant effect of lag $[F(1,31)=40.12$, $p<.001]$ and a significant interaction effect between lag and experiment $[F(1,31)=5.32, p=.028]$, confirming that the magnitude of lag-1 sparing was greater in Experiment 4 than in Experiment 2. The second analysis involved the same comparison between Experiments 4 and 3 . The analysis again revealed a significant effect of lag $[F(1,33)=35.06, p<.001]$ and a significant interaction effect between lag and experiment $[F(1,33)=5.97$, $p=.02]$, confirming that the magnitude of lag-1 sparing was greater in Experiment 4 than in Experiment 3.

The outcomes of the present analyses confirm the hypothesis that the magnitude of lag-1 sparing in the different condition is greater when the two RSVP streams are balanced with respect to meaningfulness (as in Experiments 1 and 4) than when they are unbalanced (as in

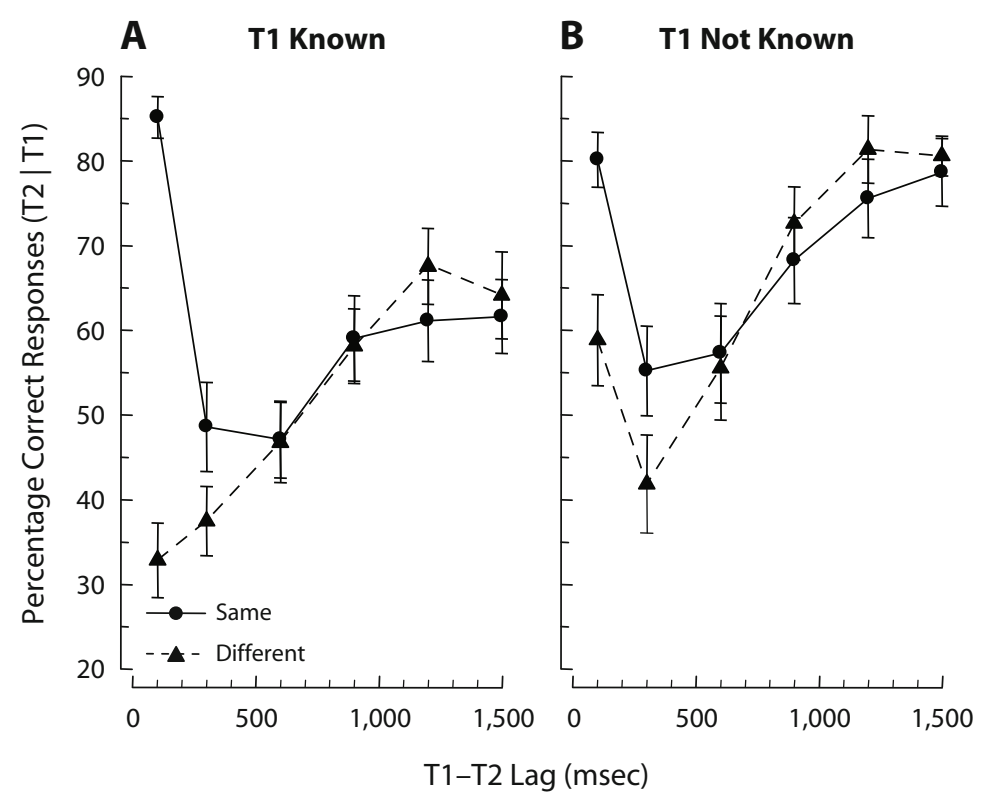

Figure 5. Mean percentages of correct identifications of the second target in Experiment 4 for those trials in which the first target was identified correctly (T2|T1). The displays consisted of two RSVP streams of random-dot patterns. In the same condition, the two letter targets were presented in the same RSVP stream; in the different condition, they were presented in opposite streams. Observers in the T1-known group (A) were given advance knowledge of the stream in which the first target would appear. Observers in the T1-not-known group (B) had no such knowledge. T1, first target; T2, second target; RSVP, rapid serial visual presentation. 
Experiments 2 and 3). In this respect, the magnitude of lag-1 sparing in the different condition of the not-known group of Experiment 1 matched that of the corresponding condition in Experiment 4. A similar correspondence was obtained between Experiments 2 and 3. This pattern of results is consistent with the hypothesis that the attentional gate can be expanded consistently to encompass both potential target locations only if the two RSVP streams are balanced with respect to meaningfulness.

An unanticipated discrepancy between the different conditions in the known groups of Experiments 1 and 4 invites comment. Accuracy of second-target identification at the two shortest lags (lags 1 and 3) was substantially lower in Experiment 4 (Figure 5A, segmented line) than in Experiment 1 (Figure 2A, segmented line). The lower performance in Experiment 4 is entirely in line with the attentional gating account presented in the Discussion of Experiment 1. Namely, in the known condition, the attentional gate was initially confined narrowly to the firsttarget stream but, upon the arrival of the first target, had to be widened to encompass both streams. On the evidence provided by Weichselgartner and Sperling (1987), this change in attentional focus would have taken about $300 \mathrm{msec}$, causing items in the other stream - notably the second target - to be missed.

Although it accounts for the relatively low secondtarget performance at lag 1 in the different condition of Experiment 4, this reasoning cannot account for the relatively high level of performance in the corresponding condition in Experiment 1 (Figure 2A, segmented line). One plausible account can be couched in terms of the relative width of the attentional gate in Experiments 1 and 4. It is possible that the gate might have been set more widely in Experiment 1 than in Experiment 4 because the meaningful items in Experiment 1 might have prevented the gate from being set narrowly on the first-target stream to the exclusion of the other stream. This would have allowed items in the other stream partial access to the gate, with consequently greater accuracy of second-target identification. Some evidence consistent with this interpretation is provided by the finding that accuracy of first-target identification was significantly higher in the different condition of the known group in Experiment 4 than in Experiment 1 $[F(1,32)=7.08, p=.012]$. This attests to the greater concentration of attention on the first-target stream in Experiment 4. It goes without saying that this account is entirely speculative and, therefore, in need of empirical verification.

\section{GENERAL DISCUSSION}

The primary objective of the present work was to resolve an apparent inconsistency in the AB literature-namely, whether lag-1 sparing occurs when the two targets are presented in different spatial locations. Lag-1 sparing denotes the finding, reported in about half of the $A B$ experiments, that the second-target deficit does not occur - or is much reduced - when the second target is presented directly after the first. A meta-analysis by Visser, Bischof, and Di Lollo (1999) revealed that lag-1 sparing never occurs when the two sequential targets are presented in different locations. An exception to this rule was reported by Shih (2000), who found substantial lag-1 sparing when the two targets were presented in different locations.

A comparison of Shih's (2000) study with the studies reviewed by Visser, Bischof, and Di Lollo (1999) revealed two key factors that might account for the discrepancy in the results: the observer's advance knowledge of the location of the first target and the number of RSVP streams in the display. The present work demonstrates that the first factor, knowledge of the first target's location, determines whether lag-1 sparing occurs when the two targets are presented in different locations. It further shows that the second factor, number of RSVP streams, is not in itself a direct determinant of lag-1 sparing. Rather, it mediates the magnitude of lag-1 sparing by determining the extent to which the two RSVP streams are balanced in the degree to which they capture attention.

In the first of four experiments, observers viewed two RSVP streams of digit distractors presented one on either side of fixation. Two letter targets could appear either in the same or in opposite streams. One group of observers knew in advance in which stream the first target would appear; a second group had no such knowledge. The results replicated the conventional finding that lag-1 sparing invariably occurs when the two targets are presented in the same location (Visser, Bischof, \& Di Lollo, 1999) as well as Shih's (2000) finding of lag-1 sparing when the targets appear in different locations. The outcome of Experiment 1 built on Shih's finding by showing that when the targets are presented in different locations, lag-1 sparing occurs only if the observer has no advance knowledge of where the first target will appear.

The results of Experiment 1 as well as those of Shih (2000) and Visser, Bischof, and Di Lollo (1999) can be explained within a conceptual framework based on a dynamically adjustable attentional gate. In this gating model, the spatial extent of the gate is said to be dynamically adjustable so as to optimize performance on the task at hand. In the discussion of Experiment 1 we described how this gating model can explain why lag-1 sparing occurs when the two targets appear in different locations, but only if the observer has no advance knowledge of the location of the first target. This is because in the absence of knowledge of the first target's location, the attentional gate must be set broadly so as to encompass both potential target locations. Given this wide setting, both targets will pass through the gate whether they are presented in the same or in different locations, and lag-1 sparing will ensue. By the same token, lag-1 sparing will not occur when the observer has advance knowledge of the first target's location because the gate is centered narrowly on the first target's location and, therefore, does not encompass the location on the opposite side of fixation. If the second target then appears at the latter location, it will not pass through the gate and lag-1 sparing will not occur.

Predictions from this model were verified in Experiments 2, 3, and 4, which confirmed the dependence of lag-1 sparing on the observer's advance knowledge of the first target's location. In addition, the results revealed that 
the magnitude of lag-1 sparing when the two targets appeared in different locations and the location of the first target was not known, is predicated on the interplay of two conflicting factors: a tendency to expand the attentional gate to encompass both potential target locations, and an opposing tendency to focus narrowly on the stream that contains the more meaningful stimuli. Thus, the magnitude of lag-1 sparing was greater in Experiments 1 and 4, in which the two streams were balanced with respect to meaningfulness (in Experiment 1, both were meaningful streams of digits; in Experiment 4, both were meaningless streams of random-dot patterns), than in Experiments 2 and 3 , in which meaningfulness was unbalanced across the two streams (Experiment 2 contained one meaningful stream of digits and one blank location; Experiment 3 contained one meaningful stream of digits and one meaningless stream of random-dot patterns).

\section{Concluding Comments}

The primary objective of the present work was to resolve an apparent inconsistency in the attentional blink literature regarding the occurrence of lag-1 sparing when the two targets are presented in different spatial locations (Shih, 2000; Visser, Bischof, \& Di Lollo, 1999). Two factors emerged as joint determinants of the incidence and magnitude of lag-1 sparing. The first factor, the observer's advance knowledge of the first target's location, determines whether lag-1 sparing will occur when the two targets are presented in different spatial locations. The second factor, the degree to which the RSVP streams are balanced with respect to meaningfulness, determines the magnitude of lag-1 sparing. It is the interplay between these two factors that provides a resolution of the apparent inconsistency in the literature.

\section{AUTHOR NOTE}

This work was supported by a Canada Graduate Scholarship from the Natural Sciences and Engineering Research Council of Canada to L.N.J., a Postgraduate Scholarship from the Natural Sciences and Engineering Research Council of Canada to S.G., grants from the Japan Society for the Promotion of Science to J.K., and a Discovery Grant from the Natural Sciences and Engineering Research Council of Canada to V.D.L. The authors thank Mark Nieuwenstein and an anonymous reviewer for helpful comments on an earlier draft of the manuscript. Correspondence related to this article may be sent to L. N. Jefferies, Department of Psychology, University of British Columbia, 2136 West Mall, Vancouver, BC, V6T 1 Z4 Canada (e-mail: ljefferies@psych.ubc.ca).

\section{REFERENCES}

Breitmeyer, B. G. (1984). Visual masking: An integrative approach. New York: Oxford University Press.

Chun, M. M., \& Potter, M. C. (1995). A two-stage model for multiple target detection in rapid serial visual presentation. Journal of Experimental Psychology: Human Perception \& Performance, 21, 109-127.

Dell'Acqua, R., Pascali, A., Joliceur, P., \& Sessa, P. (2003). Four-dot masking produces the attentional blink. Vision Research, 43, 35-40.

Di Lollo, V., Kawahara, J.-I., Ghorashi, S. M. S., \& Enns, J. T. (2005). The attentional blink: Resource depletion or temporary loss of control? Psychological Research, 69, 191-200.

Holländer, A., Corballis, M. C., \& Hamm, J. P. (2005). Visual-field asymmetry in dual-stream RSVP. Neuropsychologia, 43, 35-40.

JeFFERIES, L. N., \& Di Lollo, V. (2006). Linear changes in the spatial extent of the focus of attention across time. Manuscript submitted for publication.
Joliceur, P., \& Dell'Acqua, R. (1998). The demonstration of shortterm consolidation. Cognitive Psychology, 36, 138-202.

Juola, J. F., Botella, J., \& Palacios, A. (2004). Task- and locationswitching effects on visual attention. Perception \& Psychophysics, 66, 1303-1317.

KAWAHARA, J. (2003). Mandatory processing of distractors: Another determining factor for the attentional blink. Japanese Psychological Research, 45, 140-151.

Kristuansson, A., \& Nakayama, K. (2002). The attentional blink in space and time. Vision Research, 42, 2039-2050.

Peterson, M. S., \& Juola, J. F. (2000). Evidence for distinct attentional bottlenecks in attention switching and attentional blink tasks. Journal of General Psychology, 127, 6-26.

Potter, M. C., Chun, M. M., Banks, B. S., \& Muckenhoupt, M. (1998). Two attentional deficits in serial target search: The visual attentional blink and an amodal task-switch deficit. Journal of Experimental Psychology: Learning, Memory, \& Cognition, 24, 979-992.

Raymond, J. E., Shapiro, K. L., \& Arnell, K. M. (1992). Temporary suppression of visual processing in an RSVP task: An attentional blink? Journal of Experimental Psychology: Human Perception \& Performance, 18, 849-860.

ShapIRo, K. L., \& RAYMOND, J. E. (1994). Temporal allocation of visual attention: Inhibition or interference? In D. Dagenbach \& T. H. Carr (Eds.), Inhibitory processes in attention, memory, and language (pp. 151-188). San Diego: Academic Press.

Shapiro, K. L., Raymond, J. E., \& Arnell, K. M. (1994). Attention to visual pattern information produces the attentional blink in RSVP. Journal of Experimental Psychology: Human Perception \& Performance, 20, 357-371.

SHIH, S. I. (2000). Recall of two visual targets embedded in RSVP streams of distractors depends on their temporal and spatial relationship. Perception \& Psychophysics, 62, 1348-1355.

Theeuwes, J., \& Burger, R. (1998). Attentional control during visual search: The effect of irrelevant singletons. Journal of Experimental Psychology: Human Perception \& Performance, 24, 1342-1353.

Visser, T. A. W., Bischof, W. F., \& Di Lollo, V. (1999). Attentional switching in spatial and non-spatial domains: Evidence from the attentional blink. Psychological Bulletin, 125, 458-469.

Visser, T. A. W., Bischof, W. F., \& Di Lollo, V. (2004). Rapid serial visual distraction: Task-irrelevant items can produce an attentional blink. Perception \& Psychophysics, 66, 1418-1432.

Visser, T. A. W., Zuvic, S. M., Bischof, W. F., \& Di Lollo, V. (1999). The attentional blink with targets in different spatial locations. Psychonomic Bulletin \& Review, 6, 432-436.

Weichselgartner, E., \& Sperling, G. (1987). Dynamics of controlled visual attention. Science, 238, 778-780.

YANTIS, S., \& Jonides, J. (1984). Abrupt visual onsets and selective attention: Evidence from visual search. Journal of Experimental Psychology: Human Perception \& Performance, 10, 601-621.

YANTIS, S., \& Jonides, J. (1996). Attentional capture by abrupt onset: New perceptual objects or visual masking? Journal of Experimental Psychology: Human Perception \& Performance, 22, 1505-1513.

\section{NOTES}

1. A $2 \times 2 \times 6$ ANOVA was performed on first-target accuracy scores with two within-subjects factors (lag: 1, 3, 6, 9, 12, and 15; and relative second-target location: same or different) and one between-subjects factor (known or not known). The analysis revealed significant main effects of lag $[F(1,31)=15.7, p<.001]$ and knowledge $[F(1,31)=17.86, p<$ $.001]$. The interaction between lag and second-target location was also significant $[F(1,31)=5.72, p=.023]$. No other effects were significant. The effect of lag is well established in the literature. It refers to the finding that first-target performance is relatively low at the shorter lags and improves at the longer lags. This is thought to be due to processing and memory loads when the two targets are presented in close temporal succession. The effect of knowledge was expected on the grounds that if the observers knew where the first target would appear, their attention would be deployed to that location, and first-target performance would be correspondingly enhanced.

The interaction effect was driven entirely by performance at lag 1 , where accuracy of first-target identification was lower in the same condition $(58.9 \%)$ than in the different condition $(69.5 \%)$. The less-accurate 
performance in the same condition can be explained on the principle that the strength of masking is related directly to the degree of similarity between the target and the mask (Breitmeyer, 1984). At lag 1 of the present experiment, the first target was followed (masked) by another letter (high similarity, stronger masking) in the same condition but by a digit (lower similarity, weaker masking) in the different condition. This interpretation is buttressed by the fact that, when lag 1 is excluded from the analysis, the relative second-target location $\times$ lag interaction is no longer significant $[F(4,124)=1.37, p=.247]$.

2. Holländer, Corballis, and Hamm (2005) have reported a study similar to the not-known condition in the present Experiment 1. It is interesting to note that in the study reported by Holländer et al. an $\mathrm{AB}$ deficit was found only when the first target was presented in the left visual field (LVF) and the second target in the right visual field (RVF). When the fields were reversed, the AB deficit failed to materialize.

To check whether this pattern of results also occurred in the present study, we reanalyzed the results of the not-known condition of Experiment 1 (which corresponded to the conditions in the study of Holländer et al., 2005), separately for when the first target was presented in the LVF and in the RVF. Specifically, the ANOVA design was a 2 (first target in LVF or in RVF) $\times 6$ (lag) factorial. The results revealed a significant effect of lag $[F(5,110)=16.81, p<.001]$, confirming the presence of an overall $\mathrm{AB}$ deficit. The effect of visual field was also significant $[F(1,22)=16.47, p<.001]$, indicating that accuracy of second-target identification was greater when the first target was in the RVF. This is the opposite of what was found by Holländer et al. Notably, the interaction effect was not significant $[F(5,110)=1.39, p=.233]$, indicating that an $\mathrm{AB}$ deficit occurred equally in both visual fields. As noted above, Holländer et al. found an $\mathrm{AB}$ only when the first target was presented in the left visual field. The reasons for this discrepancy are not immediately obvious, and warrant further investigation.

To check on the incidence of lag-1 sparing, we performed an additional analysis confined to the two shortest lags. The analysis was a 2 (first target in LVF or in RVF) $\times 2$ (lags 1 and 3 ) ANOVA. It revealed a significant effect of visual field $[F(1,22)=12.22, p=.002]$, consistent with greater second-target accuracy when the first target was in the RVF, and a significant effect of lag $[F(1,22)=33.05, p<.001]$, indicating the presence of lag-1 sparing. Notably, the interaction effect was not significant $[F(1,22)=0.86, p=.360]$, confirming that the magnitude of lag-1 sparing did not differ between LVF and RVF.

(Manuscript received March 9, 2005; revision accepted for publication March 16, 2007.) 